\title{
LES POLITIQUES ACTIVES DU MARCHE DE TRAVAIL ET LA MAIN D'OEUVRE FEMININE EN TURQUIE
}

\author{
Yrd. Doç. Dr. Berrin Ceylan Ataman \\ Ankara Üniversitesi \\ Siyasal Bilgiler Fakültesi
}

\section{Türkiye'de Aktif İsgücü Piyasası Politikalan ve Kadın Emeği Özet}

1970'li yullarda Keynesci politikaların terkedilmesi ve neo-liberal politikaların benimsenmesi isssizliği arturıa bir rol oynamıştır. Devletin yeni istihdam olanakları yaratmadaki rolünün azaldığ bu dönemde, işücü piyasası ile bağlann güçlendirilmesinde aktif politikalara yer verilmektedir. Işgücünün eğitimine yönclik olan aktif politikalardan bu piyasada istihdam sorunlu olan -kadınların, gençlerin, engellilerin, göçmenlerin vs...- yararlanmalan hedeflenmektedir. Bu çalışma aktif işü̈cü piyasası politikalarının Türkiye'de uygulanışını kadın işgücü çerçevesinde ortaya koymaktadır.

\section{Active Labor Market Policies and Feminine Labor Force in Turkey Abstract}

In 1970's the Keynesian policies have been abandoned in favor of the neo-liberal policies and this policy switch has played a role in increasing the level of unemployment. In this period, the role of the government in creating employment opportunities has decreased and active policies are used in order to strenghten the bonds between the unemployed and the labor market. The active policies designed to educate the labor force are mainly aimed at the groups which are disavantaged such as women, youngsters or the disabled. In this essay the active labor policies used in Turkey are studied within the framework of feminine labor. 


\section{Les Politiques Actives du Marché de Travail et la Main d'Oeuvre Féminine en Turquie}

\section{Introduction}

Depuis les décisions dites du 24 Janvier 1980 avec la mise en oeuvre de politiques néo-libérales en Turquie suivant la tendance de plus en plus dominantes dans le monde entier, on a pu constater que le chómage structurel ${ }^{1}$ devient un problème croissant. Le mode de production, caracterisé par l'automation et l'informatisation, a changé la demande de travail aussi bien du point de vue de la quantité que de la qualité: Le changement de mode de production par l'introduction des nouvelles techonologies a nécessité moins de main d'oeuvre, mais a exigé plus de qualifications. Par conséquent la situation des chmeurs sans qualifications a été aggravé et ceci a cré un chômage chronique caractérisé de longue durée.

Il est évident que, dans la lutte contre le chômage, les politiques économiques Keynesienne qui visent à créer de l'emploi sont nécessaires considerant que le chômage n'est pas la faute de l'individu comme le prétendaient les néo-classiques. Mais depuis les années de 1970 où les politiques Keynesienne ont été abandonnées et les politiques néo-libérales ont repris de la force, la lutte contre le chômage a changé. Car le point de vue néo-libérale exclu le rôle de l'état quant à la résolutoin du chômage. Le néo-libéralisme voit la résolution du problème de chômage dans le fonctionnement libre du marché de travail.

Dans le processus de la mondialisation qui exige la compétition et le rétablissement d'un niveau d'emploi socialement acceptable, l'éducation et la formation jouent un rôle détérminant. Ainsi l'accent est mis sur les politiques actives.

1 Le chômage structurel est défini d'une manière générale comme étant une inadaptation des conditions de l'offre de main d'ocuvre par rapport à la demande de main d'oeuvre. 
Cet article a pour but de traiter les politiques actives du marché de travail du point de vue du travail féminin en Turquie. Le choix spécifique de la main d'oeuvre féminine peut s'expliquer par le fait que; la majorité des politiques actives mises en oeuvre en Turquie est orientée vers les femmes. Car d'une part, la main d'oeuvre féminine se porte plus ce mode de travail et que l'entrepreneur économisant d'avantage sur les cotes de production cela lui permet d'augmenter sa compétition. D'autre part, le travail des femmes -plus particulièrement dans les milieux urbains-, bien qu'il soit moins cher ou qu'il soit dans des conditions informelles, constitue une contribution considérable au revenu du ménage. En plus la femme peut travailler chez elle, ce qui rend tolérable le travail des femmes même dans les milieux conservateurs (DEMIREL, 1999: 13-15).

Pour mieux exposer ce problème nous allons d'abord expliquer ce qu'on entend par la politique active. Nous aborderons ensuite brièvement les caractéristiques du marché de travail féminin en Turquie. Finalement le texte sera consacré l'analyse des conséquences de la mise en oeuvre des politiques actives en faveur de la main d'ocuvre féminine.

\section{1- Définition de la Politique Active}

La politique active est définie comme étant des programmes d'éducation stimulant l'employabilité de la force de travail (BENUS, 1996). L'objectif est d'accroître l'emploi et l'employabilité des chomeurs. Les programmes de formation, d'offre d'orientation professionnelle, de développement des stratégies de recherche d'emploi... sont souvent spécifiques aux groupes tels que: Les jeunes, les femmes, les handicapés, les immigrés...

La formation, clé de la politique active du marché de travail, sert adapter les qualifications de la main d'oeuvre aux besoins du marché, par conséquent c'est l'un des éléments de la flexibilité du marché du travail. L'objectif est de faciliter l'insertion des jeunes et des femmes sur le marché du travail. (COMMISSION EUROPEENNE, 1994: 138)

Les politiques actives du marché du travail --contrairement aux politiques passives-- exigent une certaine élasticité du point de vue des droits sociaux. Par exemple l'assurance chômage qui est un instrument de la politique passive du marché du travail est bien défini par les lois, donc les conditions permettant de bénéficier de cette assurance ont force de loi. Or, l'intervention par les politiques actives sur le marché de travail dépend des décisions prises par l'état donc de décisions caractère politique. Une fois la décision politique prise, l'institution concernée s'engagera effectuer le programme et détérminer la population qui sera ciblée par le programme.

Les pays en voie de développement ne sont en mesure d'entrer dans la 
concurrence que dans les secteurs où le facteur de la main d'oeuvre est déterminant dans la production comme dans le cas du textile. ${ }^{2}$ Le mode de production flexible a rendu la main d'oeuvre féminine de plus en plus attractive car elles constituent la main d'oeuvre la moins chére et elles sont prêtes à travailler dans le secteur informel. C'est la raison pour laquelle les politiques actives visent plutôt les femmes.

\section{II- Les Caracteristiques du Travail Feminin en Turquie}

Le taux de participation à l'activité économique des femmes est le principal indicateur faisant apparaître les traits essentiels de la main d'oeuvre féminine. Le taux de participation féminine change suivant le niveau de développement économique. Plus l'économie est organisée autour du secteur agricole, plus la participation féminine est forte. En effet, la force de travail féminin est abondante dans l'agriculture. A l'issue de l'industrialisation qui provoque à son tour l'urbanisation, la femme reste de plus en plus à la maison pour accomplir le rôle traditionnellement réservé à son sexe tel que le ménage, l'éducation des enfants etc... Mais il y a aussi une corrélation évidente entre le niveau d'éducation et le taux de participation a l'activité économique. Du point de vue historique en Turquie, le taux de participation total est en légère diminution, ce qui s'explique, en grande partie, par l'augmentation générale du niveau d'éducation.

Tableau 1 Le taux de particlpalion (\%) (1989-2000)

\begin{tabular}{|l|c|c|c|c|c|c|c|c|c|}
\hline Année & \multicolumn{3}{|c|}{ Turquic } & \multicolumn{3}{c|}{ Urbain } & \multicolumn{3}{c|}{ Rural } \\
& Total & Femmes & Hommes & Total & Femmes & Hommes & Total & Femmes & Hommes \\
\hline 1989 & 55.3 & 35.1 & 75.7 & 44.5 & 16.4 & 71.8 & 66.8 & 54.4 & 79.9 \\
1990 & 52.6 & 31.8 & 73.8 & 43.0 & 15.1 & 70.3 & 63.0 & 49.2 & 77.7 \\
1991 & 53.9 & 33.2 & 74.9 & 42.9 & 15.3 & 70.0 & 65.9 & 52.2 & 80.5 \\
1992 & 51.6 & 30.6 & 73.0 & 43.3 & 15.7 & 70.4 & 61.0 & 46.9 & 76.9 \\
1993 & 50.3 & 29.9 & 71.0 & 41.6 & 15.0 & 67.7 & 60.4 & 46.5 & 75.1 \\
1994 & 51.0 & 31.1 & 71.1 & 42.0 & 16.0 & 67.6 & 61.6 & 48.5 & 75.4 \\
1995 & 50.4 & 30.3 & 70.7 & 41.0 & 14.9 & 66.6 & 61.8 & 48.4 & 75.9 \\
1996 & 50.0 & 30.1 & 70.2 & 40.6 & 14.6 & 66.2 & 61.6 & 48.6 & 75.2 \\
1997 & 48.4 & 27.9 & 69.2 & 39.3 & 14.5 & 64.4 & 60.0 & 45.0 & 75.5 \\
1998 & 47.5 & 26.4 & 69.0 & 39.8 & 14.9 & 64.7 & 57.9 & 41.5 & 74.9 \\
1999 & 48.7 & 29.7 & 68.3 & 39.9 & 15.8 & 64.3 & 60.3 & 47.6 & 73.7 \\
$2000^{*}$ & 46.7 & 23.7 & 69.8 & 43.0 & 16.9 & 69.2 & 52.4 & 34.3 & 70.8 \\
\hline
\end{tabular}

Source: DIE 1999

* Les données de l'an 2000 concernent la première partie de l'année

2 Par exemple en Turquie l'exportation des produits textile a triplé en 1992 par rapport à l'année 1972 (ERAYDIN 1997: 7 ). 
Le tableau ci-dessus montre que le taux de participation féminine est supérieur dans les régions rurales par rapport à celui des zones urbaines. Cela s'explique, d'une part, par des facteurs culturels qui constituent des obstacles sérieux à la participation des femmes au travail dans les villes, parce que l'exode rural fait que les femmes ne travaillent pas dans les villes, elles accomplissent leurs taches dite féminines; d'autre part, par des facteurs économiques, car l'industrialisation ne crée pas assez d'emploi, d'où il y a un vrai problème de chômage.

L'analyse du problème de chômage en Turquie pourrait être étudié sous deux aspects: Le chômage et le sous emploi. Ainsi le chômage n'est pas seulement lié au probleme de recherche d'emploi ou au manque d'emplois mais surtout un problème d'embauche. Les employés sont souvent embauchés aux travaux qui ne correspondent pas à leurs professions, des salaires très bas dans plusieurs secteurs et souvant sans sécurité sociale. Par conséquent, ils sont considérés comme des ouvriers intérimaires.

En Turquie le taux de sous emploi est de $6.9 \%$. I est de $8.8 \%$ chez les hommes et de $2.5 \%$ seulement chez les femmes. Le sous emploi est beaucoup plus important parmi les jeunes diplomés soit $9.6 \%$ total dont $12.5 \%$ chez les hommes et $5.6 \%$ chez les femmes. Ces données indiquent que le sous emploi est beaucoup plus important pour les hommes que pour les femmes.

En Turquie le taux de chomage est de 7.3\%: $6.4 \%$ chez les femmes et $7.7 \%$ chez les hommes. Le taux de chômage féminin est légèrement inférieur celui des hommes. Mais il faut remarquer qu'il n'est pas facile de déterminer le travail féminin en quantité, car il est essentiellement executé sans rémunération. Par contre dans les villes, le chômage est beaucoup plus important chez les femmes que chez les hommes, soit de $16.4 \%$ contre $10.6 \%$. Car les femmes travaillent sans être déclarées dans le secteur informel. Mais cela ne depend pas d'elles; si elles avaient le choix, elles préfereraient trouver un emploi dans le secteur formel.

Les politiques actives visent a augmenter et a faciliter la participation des femmes à la force de travail. La corrélation entre le niveau d'éducation et celui de chômage devient un point clé: 
- Ankara Universitesi SBF Dergisi • 55-4

\section{Tableau 2}

Le taux de chômage selon le niveau d'éducation (en millier) 1999

\begin{tabular}{|c|c|c|c|c|c|c|c|}
\hline & $\begin{array}{c}\text { L a force de } \\
\text { travail }\end{array}$ & $\begin{array}{c}\text { Chômage } \\
\text { total }\end{array}$ & $\%$ & $\begin{array}{l}\text { Chômage } \\
\text { des femmes }\end{array}$ & $\%$ & $\begin{array}{c}\text { Chômage } \\
\text { des hommes }\end{array}$ & $\%$ \\
\hline Total & 23779 & 1730 & 7.3 & 471 & 6.4 & 1259 & 7.7 \\
\hline Illitré & 2223 & 48 & 2.1 & 12 & 0.8 & 35 & 4.9 \\
\hline Littré sans diplôme & 904 & 41 & 4.5 & 7 & 2.5 & 33 & 5.4 \\
\hline Ecole primaire & 13074 & 759 & 5.8 & 146 & 3.9 & 614 & 6.6 \\
\hline $\begin{array}{l}\text { Ecole secondaire } \\
\text { (1er cycle) }\end{array}$ & 2378 & 227 & 9.5 & 46 & 12.9 & 181 & 8.9 \\
\hline $\begin{array}{l}\text { Ecole secondaire de } \\
\text { profession } \\
\text { (1er cycle professionnelle) }\end{array}$ & 50 & 10 & 19.2 & 2 & 67.1 & 7 & 15.9 \\
\hline Lycée & 2494 & 403 & 16.1 & 155 & 22.7 & 248 & 13.7 \\
\hline Lycée professionnel & 938 & 130 & 13.9 & 54 & 25.4 & 76 & 10.5 \\
\hline $\begin{array}{l}\text { Ecole supérieure ou } \\
\text { Université }\end{array}$ & 1717 & 113 & 6.6 & 48 & 8.8 & 64 & 5.5 \\
\hline
\end{tabular}

Source: DIE 1999

La conclusion la plus évidente à tirer du tableau ci-dessus est que le chômage féminin atteint un niveau elevé pour la catégoric détentrice d'un diplôme de lycéc. Cela peut s'expliquer en dehors des motifs culturels, par le fait que l'enseignement secondaire ne donne pas de qualification suffisante pour trouver un travail, autrement dit la formation professionelle est insuffisante d'où un grand problème d'adaptation au marché du travail. Une autre raison très importante est que de nombreux emplois requérant une formation du niveau de baccalauréat sont occupés par des diplomés d'université qui n'ont pu trouver un emploi à leur niveau. Problème du sous emploi d'une partie des diplomés d'université qui, faute de trouver un emploi à leur niveau de compétence ont $d \hat{a}$ accepter un poste inférieur dont nous avons déj à parlé ci-dessus.

Le fait que l'industrialisation ne soit pas développée suffisamment pour créer assez d'emploi dans les grandes villes, le secteur informel ${ }^{3}$ s'est développé et il a absorbé la main d'ocuvre féminine. Les caractéristiques traditionelles (la

3 Le sectuer informel se définit par les relations avec l'état; les liaisons sistématiques avec l'économie formelle et les caractéristiques de la force de travail. 
structure archaique de la famille, la répartition du travail déterminé par le sex etc...) ont poussé les femmes dans le secteur informel. L'évolution de la production a entrainé la création de taches réalisables à la maison ce qui arrangeait aussi bien les industries que les femmes. Bien qu'on ne dispose pas de chiffres officiels on estime qu'il $y$ a autant de femmes qui travaillent dans le secteur informel que dans le secteur formel. L'existence de cette force de travail féminine définie comme "non-qualifiée" et "moins chère" entraîne la création d'emploi féminins.

Les recherches éffectuées ces dérnières années montrent que le secteur informel n'est pas marginal, mais entièrement integré dans l'économie. Les grandes firmes ont décentralisé leurs productions et leur réseaux de distributions en utilisant souvent les forces informelles (FRETWELL, 1998). Sur le marché du travail urbain, la majorité sont des salariés. Le problème essentiel qui y existe est le bas salaire qui provoque la naissance du secteur marjinal ou le secteur informel; ces derniers servent à recompenser le problème du bas salaire.

La caractéristique la plus déterminante du marché de l'emploi est la répartition des statuts des travailleurs.

\section{Tableau 3}

La répartition selon les catégories d'emploi (en millier) 1999

\begin{tabular}{|l|c|c|c|c|c|c|}
\hline & Total & $\begin{array}{c}\text { Employé } \\
\text { régulière }\end{array}$ & $\begin{array}{c}\text { Employé } \\
\text { saisonnier }\end{array}$ & Entreprencur & $\begin{array}{c}\text { Travailleur } \\
\text { son prorpre } \\
\text { compte }\end{array}$ & $\begin{array}{c}\text { Travailleur } \\
\text { sans } \\
\text { rémunération }\end{array}$ \\
\hline Total & 22049 & 7143 & 1901 & 1019 & 5463 & 6523 \\
Femmes & 6882 & 1447 & 291 & 34 & 633 & 4477 \\
Hommes & 15167 & 5696 & 1610 & 985 & 4831 & 2046 \\
\hline
\end{tabular}

Source: DIE 1999

Le tableau ci-dessus indique que dans le total de la main d'oeuvre féminine seules $21.0 \%$ occupent un emploi régulier, $65.0 \%$ sont des travailleurs de famille sans rémunération. $\mathrm{Si}$ on observe la situation en milieu rural, on constate que le chiffre des travailleurs sans rémunération atteint un niveau impressionnant: Parmi 5030000 femmes employées dans le milicu rural 4198 000 sont des travilleuses sans rémunération soit $83.3 \%$. C'est un aspect très important du marché du travail en Turquie. 


\section{III- Les Politiques Actives Mise en Oeuvre en Faveur de la Main d'Oeuvre Feminine en Turquie}

En Turquie le début des politiques actives date de 1988. En 1993 l'Agence Nationale Pour Emploi (ANPE) turque qui est résponsable de ces politiques a reçu des crédits considérables de la Banque Mondiale ce qui a permis un développement en ce qui concerne la formation de la main d'oeuvre en général (TISK, 2000).

Les politiques actives poursuivies en Turquie peuvent être structurées en deux parties

\section{1- Les cours organisés par le ministre de l'éducation:}

Ces cours constituent la composante essentielle des activités de formation professionnelle. Ils sont orientés plus particulièrement vers la main d'ocuvre féminine. En effet presque $90 \%$ des apprenants sont des femmes. La grande majorité des activités de formation professionnelle consistent en des écoles des arts pratiques pour les jeunes filles.

Les programmes d'apprentissage et les centres de formation sont les principales institutions du Ministre pour propager l'éducation au niveau de la population. Les centres de formation qui existent un peu partout travaillent comme un établissement de réhabilitation: On peut en trouver dans les villages, dans les prisons, dans les hôpitaux, dans les lieux de travail privé et/ou public etc... Ces centres ouvrent des cours pour les analphabètes ou bien des cours de formation professionnelle. Tous les ans, des cours portant sur 120 branches de métiers sont organisés. Ce sont en grande majorité des cours consacrés à la formation de la main d'ocuvre féminine. Tel que: couture, nappage, tapisserie, etc... (AKHUN, 1999).

En ce qui concerne les programmes d'apprentissage, ils sont également orientés vers les femmes. Il existe aussi des institutions formant dans le domaine commercial et le tourisme d'éducation; centre d'éducation technique des filles.

\section{2- Les cours de formation organisés par I'ANPE turque (Le Ministère du Travail)}

Les activités réalisćes par l'ANPE turque consistent essentiellement en des programmes de formation professionnelle tendant à la stabilité de l'emploi. Ces cours de 3 a 6 mois qui ont commencé le 11 Janvier 1988, ont contribué de manière importante à la lutte contre le chômage. Les programmes de formation professionnelle qui sont organisés par l'ANPE visent plutôt les chomeurs jeunes, non qualifiés et de longue durée, comme c'est les cas dans les pays de l'OCDE et dans ceux de l'UE (COMMISSION EUROPEENNE, 1994). D'après les analyses 
Berrin Ceylan Ataman • Les Politiques Actives du Marché de Travail et la Main d'Oeuvre Féminine en Turquie $\bullet \mathbf{9}$

faites par la direction chargée des affaires et du statut de la femme, on observe les résultats suivants (AKHUN, 1999; DEMIREL, 1999):

- D'après les données, le nombre des apprenants augmentent d'une année sur l'autre et en moyenne $83.7 \%$ des apprenants finissent les cours et environ $82.7 \%$ trouvent un emploi. Les femmes constituent $61 \%$ des participants. Il s'agit plutôt de professions liées au tourisme, au textile, à la confection, à l'informatique, à la tapisserie et à la manufacture.

- Ces programmes sont effectués par l'ANPE et aussi par quelques établissements privés, dans les régions industrialisées. Les programmes visent les personnes qui ont un certain niveau d'éducation mais à qui manquent une formation répondant aux besoins du marché du travail. Autrement dit ces programmes apportent des solutions au problème du rapport existant entre la qualité et la quantité de travail offert (DPT, 1999).

- En général ces cours sont plus nombreux dans les grandes villes industrialisées et ce sont plutôt les jeunes et les femmes qui s'y intéressent. Dans les régions rurales il existe des cours plus spécifiques qui visent plutôt l'artisanat.

Il existe également des cours de formation avec garantie d'emploi organisé par l'ANPE. Les apprenants ont participé à ces cours, persuadés qu'il auraient un métier et ils seraient employés plus facilement. Les apprenantes (les femmes) ont marqué plus d'intêret pour les formations entraînant un emploi dans la fonction publique, faisant plus confiance à ce secteur en ce qui concerne la sécurité de l'emploi et les garanties sociales. Selon une enquête éffectuée par l'ANPE (AKHUN, 1999) sur les "programmes de formation avec garantie d'emploi" il est à noter que $82.7 \%$ des participants sont célibataires et en majorité des femmes. Ceci s'explique par le fait que les femmes préfèrent obtenir leurs émancipations avant d'être mariées. Par contre les femmes mariées avec un ou plusieurs enfants participent moins à ce genre d'activités car leurs rôles sont bien définis dans la société.

La formation professionnelle permet aux apprenants d'obtenir une garantie de l'emploi mais la durée de travail est en moyenne de 9 mois pour les raisons suivantes:

- Pendant la période d'emploi ils ont des relations avec d'autres entreprises ou bien ils font la connaissance du milicu de travail, qui leur permet de changer "pour un meilleur emploi". Autrement dit pour les apprenants ces cours sont un bon tremplin pour l'accès au marché du travail et aussi un espoir de trouver par la suite un meilleur emploi. 
- La plupart ne sont pas très satisfaits de leurs salaires (les salaires sont très bas en général dans le pays) et de leurs conditions de travail (très souvent non déclaré).

- Les raisons de changer ou de quitter le travail changent selon le métier. Par ex. ceux qui travaillent dans linformatique et dans le textile changent de travail moins fréquemment que ceux qui travaillent dans le secteur du tourisme. ${ }^{4}$

\section{Conclusion}

Les cours de formation professionnelle qui constituent une partie importante des politiques actives sont en évolution considérable en Turquie. Les recherches montrent que la plupart des participants sont des femmes. La contribution économique des femmes est poussée par la détérioration du niveau de vie des familles; par conséquent l'émancipation vient toute seule. Ceci ouvre la voie à une augmentation de la participation de la force de travail féminin. Mais les femmes se heurtent des problemes assez importants dans leur participation aux activités de formation pour les raisons suivantes:

- Premièrement les femmes ne peuvent participer à ces cours qu'avec le consentement de leurs parents ou si elles sont mariées de leurs maris. Plus de la moitié des apprenantes mariées ont un enfant. L'éducation des enfants étant considérée comme une tache appartenant à la mère, celle-ci doit trouver l'aide d'un parent pour obtenir le soutien de son mari à sa participation à cette formation.

- Celles qui participent aux cours de formation sont en majorité des jeunes de 18-24 ans. Ce qui nous montre que la jeune génération féminine est plus sensible à son émancipation.

- Les femmes sont formées en majorité dans le textile ou la conféction. Secteur de bas salaires et bien souvant non déclarés dans lesquels les conditions de travail sont particulièrement mauvaises. En résumé le travail des femmes n'est pas protégé par une législation, donc c'est un travail informel toujours sous la menace de licenciement.

Les politiques actives servent à faciliter l'insertion des chômeurs -plus particulièrement des jeunes et des femmes- sur le marché du travail. Le développement qualitatif de la main d'oeuvre féminine entraînera le

4 Il existe également des cours de formation professionnelle organisćs:

1- Par le Ministère de la Justices visant la formation des prisonniers,

2- Par le Ministère de l $A$ griculture visant particulièrement le jeunes des régions rurales.

3- Par le Minisère de Tourisme visant a former du personnel qualifiè dans le secteur du tourisme. 
Barria Coylan Ataman • Les Politiques Actives du Marché de Travail et la Main d'Oeuvre Féminine en Turquie • 11

changement social et économique de la société. Mais le problème du chômage ne pourra être résolu sans la création de nouvaux emplois et surtout une lutte sévère contre le secteur informel qui ne pourra se réaliser que par l'intermédiaire d'une législation stricte et rapide.

\section{Références}

AKHUN, Ihån (1999), Isgücü Yeliştime Kurslarnnın Kadin Istihdamina Katkısı (Ankara: T.C. Başbakanlık Kadının Statüsũ ve Sorunlanı Genel Müdürluğü).

BENUS. Jacob / VARÇIN. Recep (1996), Impact Analysis of Active Labor Programs: Study of Active Labor Labor Programs (Warsaw).

COMMISSION EUROPEENNE (1994), Croissance, Compétitivité, Emploi, Liure Blanc (Luxembourg).

DEMIREL. Ahmet (1999). Calısmaya Hazır Isgūcü Olarak Kentli Kadın ve Degişimi (Ankara: T.C. Başbakanlik Kadının Staüsü ve Sonunları Genel Müdürlügü).

DIE (1999), Hanehalkı Isgücü Anketleri (Ankara: T.C. Başbakanlık).

DPT (1999), 2000 Yili Programe (Ankara: T.C. Basbakanlik).

ERAYDIN, Ayda (1999), Yeni Oretim Süreçleri ve Kadının Emeği (Ankara: T.C. Başbakanlık Kadının Staüsu ve Sorunları Genel Müdürlügü).

FRETWELL. D. / BENUS, J. C. O. (1998), Evaluating the Impact of Active Labor Programs: Results of Cross Country Studies in Europe and Central Asia (World Bank Technicel Paper).

TISK (2000). Issizlik Sigortası, Aktif Islihdam Polilikalan ve Ozel Istihdam Bürolan (Ankara: Işveren Sendikalanı Konfederasyonu). 